\title{
Density for solutions to stochastic differential equations with unbounded drift
}

\author{
Christian Olivera ${ }^{1, *}$ Ciprian A. Tudor ${ }^{2}$ \\ ${ }^{1}$ Departamento de Matemática, Universidade Estadual de Campinas, \\ 13.081-970-Campinas-SP-Brazil. \\ colivera@ime.unicamp.br \\ ${ }^{2}$ Laboratoire Paul Painlevé, Université de Lille 1 \\ F-59655 Villeneuve d'Ascq, France. \\ tudor@math.univ-lille1.fr
}

May 18, 2018

\begin{abstract}
Via a special transform and by using the techniques of the Malliavin calculus, we analyze the density of the solution to a stochastic differential equation with unbounded drift.
\end{abstract}

MSC 2010: Primary 60H15: Secondary 60H05.

Key Words and Phrases: stochastic differential equations, unbounded drift, Malliavin calculus, existence of the density.

\section{Introduction}

Our purpose is to prove the existence and other properties for the density of the solution to the stochastic differential equation $(\mathrm{SDE})$ in $\mathbb{R}^{d}$

$$
X_{t}(x)=x+\int_{0}^{t} b\left(X_{r}(x)\right) d r+\int_{0}^{t} \sigma\left(X_{r}(x)\right) d B_{r}, \quad t \in[0, T], x \in \mathbb{R}^{d}
$$

with unbouded Hölder continuous drift $b$ and smooth diffusion coefficient $\sigma$.

The Malliavin calculus is nowadays a widely recognized mathematical theory to study densities of random variables in general, and of solutions to stochastic (partial) differential equations in particular. On the other hand, the theory works well when the coefficients of the stochastic equations are smooth enough. For instance, one can prove the absolute continuity with respect to the Lebesque measure of the law of solutions to SDEs with coefficients which are globally Lipschitz continuous and of at most linear growth and with invertible diffusion matrix. For more regular coefficients, e.g. for bounded functions with bounded derivatives, we can get the existence of a smooth density for the solution to (1). We refer, among others, to [22] or [26] for a more complete exposition.

On the other hand, when the coefficients are less regular, for example if they are only Hölder continuous, then much more work is needed in order to obtain the Malliavin differentiability of the

* Supported by FAPESP 2012/18739-0 
solution or the existence and the smoothness of its density. There already exists a large literature in this direction, see among others, 44, [13, [15], 24], [12, [2], [6], 19] etc. As far as we know, the study of the density for solutions to (11) in the case of unbounded Hölder continuous drift $b$ and in general dimension $d \geq 1$ is not contained in none of these references. Let us briefly discuss the closest results to our paper. For $d=1$, the absolute continuity of the solution to (10) has been showed in [11]. In [6], the existence of the density for the solution to a special one-dimensional SDE with Hölder continuous diffusion and drift with at most linear growth has been considered. Further improvements have been achieved in [2] in the general case $d \geq 1$, by weakening the Hölder continuous hypothesis. In [19, the author shows that if there exists some ball in $\mathbb{R}^{d}$ in which both coefficients are smooth and with bounded partial derivatives, then the density is smooth inside a smaller ball. In the case $d \geq 1$, [12] shows that if there exists some open interval on which $b$ is Hölder continuous, uniformly elliptic and smooth, then the density is Hölder continuous on the interval. The case of bounded Hölder continuous diffusion coefficient in dimension $d \geq 1$ is treated also in [3]. Stronger, or different, assumptions on the drift are considered in [4], 13], [15, 24].

Our purpose is to present a new and easier method to deal with densities of solutions to SDEs with irregular drift, via some special transformations of the drift and of the diffusion coefficient. The basic ideas is as follows: by using a transformation defined in [8], we can show that the equation (1) is equivalent to another SDE

$$
Y_{t}(x)=x+\int_{0}^{t} \widetilde{b}\left(Y_{r}(x)\right) d r+\int_{0}^{t} \widetilde{\sigma}\left(Y_{r}(x)\right) d B_{r}
$$

where the "new coefficients" $\widetilde{b}$ and $\widetilde{\sigma}$ are explicitly constructed from $b$ and $\sigma$. The equivalence between the equations (11) and (2) means that if (1) has a solution $X_{t}(x)$ then (2) has a solution $Y_{t}(x)$ and $X_{t}(x)=\Phi^{-1}\left(Y_{t}(x)\right)$ for every $t \in[0, T], x \in \mathbb{R}^{d}$ with $\Phi$ a smooth and invertible function. On the other hand, the new coefficients $\widetilde{b}$ and $\widetilde{\sigma}$ are smooth and the standard Malliavin techniques can be applied to (2). This will easily lead to the Malliavin differentiability of the solution to (1) and to the existence of its probability density. This is the main purpose of our work, to show that our approach allows, by relatively trivial arguments, to obtain results that usually demand significant technical work. A similar idea has been marginally employed in [25].

Our paper is organized as follows. Section 2 is devoted to the introduction of some notation and of the basic elements of the Malliavin calculus. In Section 3 we present the special transform used in our work and we derive some consequences for the Malliavin differentiability and the density of the solution. In Section 4 we analyze the existence of the density for functionals of the solution.

\section{Preliminaries}

This preliminary section is devoted to the presentation of the basic tools from Malliavin calculus and to the introduction of some notation needed in the paper.

\subsection{Malliavin derivative}

Let us present the elements from the Malliavin calculus that will be used in the paper. We refer to 22] for a more complete exposition. Consider $\mathcal{H}$ a real separable Hilbert space endowed with the scalar product $\langle\cdot, \cdot\rangle_{\mathcal{H}}$ and $(B(\varphi), \varphi \in \mathcal{H})$ an isonormal Gaussian process on a probability space $(\Omega, \mathcal{A}, \mathbb{P})$, that is, a centred Gaussian family of random variables such that $\mathbf{E}(B(\varphi) B(\psi))=\langle\varphi, \psi\rangle_{\mathcal{H}}$.

We denote by $D$ the Malliavin derivative operator that acts on smooth functions of the form 
$F=g\left(B\left(\varphi_{1}\right), \ldots, B\left(\varphi_{n}\right)\right)\left(g\right.$ is a smooth function with compact support and $\left.\varphi_{i} \in \mathcal{H}, i=1, \ldots, n\right)$

$$
D F=\sum_{i=1}^{n} \frac{\partial g}{\partial x_{i}}\left(B\left(\varphi_{1}\right), \ldots, B\left(\varphi_{n}\right)\right) \varphi_{i} .
$$

It can be checked that the operator $D$ is closable from $\mathcal{S}$ (the space of smooth functionals as above) into $L^{2}(\Omega ; \mathcal{H})$ and it can be extended to the space $\mathbb{D}^{1, p}$ which is the closure of $\mathcal{S}$ with respect to the norm

$$
\|F\|_{1, p}^{p}=\mathbf{E} F^{p}+\mathbf{E}\|D F\|_{\mathcal{H}}^{p} .
$$

We denote by $\mathbb{D}^{k, \infty}:=\cap_{p \geq 1} \mathbb{D}^{k, p}$ for every $k \geq 1$. In this paper, $\mathcal{H}$ will be the standard Hilbert space $L^{2}([0, T])$.

We will use the chain rule for the Malliavin derivative (see Proposition 1.2.4 in [22]) which says that if $\varphi: \mathbb{R} \rightarrow \mathbb{R}$ is a differentiable function with bounded derivative and $F \in \mathbb{D}^{1,2}$, then $\varphi(F) \in \mathbb{D}^{1,2}$ and

$$
D \varphi(F)=\varphi^{\prime}(F) D F .
$$

For a random variable $F$ with values in $\mathbb{R}$, we wll say that is Malliavin differentiable if all its components are in $\mathbb{D}^{1,2}$.

An important role of the Malliavin calculus is that it provides criteria for the existence of the density of a random variable. This was actually the initial motivation to construct this mathematical theory. There exist a huge number of formulas for densities of random variables in terms of the Malliavin operators, see [22] or [20] among others. Here we will use the following result: if $F$ is a random variable in $\mathbb{D}^{1,2}$ such that

$$
\|D F\|_{\mathcal{H}}>0 \text { a.s. then } F \text { admits a density with respect to the Lebesque measure }
$$

(see e.g. Theorem 2.1.3 in [22]). This is a classical result in Malliavin calculus, related to the invertibility of the Malliavin matrix, and it goes back to [27]. More recently, this link between Malliavin calculus and the probability densities of random variables has been reinforced and new interesting results have been obtained (see, among others, [2], 21], 23]).

\subsection{Notation}

Let us introduce some notation needed throughout the paper.

For any $\theta \in(0,1)$, we define the set $C^{\theta}\left(\mathbf{R}^{d} ; \mathbf{R}^{k}\right), d, k \geq 1$ of the mappings $f: \mathbf{R}^{d} \rightarrow \mathbf{R}^{k}$ such that

$$
[f]_{\theta}:=\sup _{x, y \in \mathbb{R}^{d}, x \neq y,|x-y| \leq 1} \frac{|f(x)-f(y)|}{|x-y|^{\theta}}<\infty .
$$

By $|\cdot|,\langle\cdot, \cdot\rangle$ we denote the Euclidean norm and scalar product in $\mathbb{R}^{k}$ respectively.

The space $C^{\theta}\left(\mathbf{R}^{d} ; \mathbf{R}^{k}\right)$ is a Banach space with respect to the norm

$$
\|f\|_{\theta}=\left\|(1+|\cdot|)^{-1} f\right\|_{\infty}+[f]_{\theta}
$$

where $\|\cdot\|_{\infty}$ denotes the supremum norm. It is also called in [8] as the space of "locally uniformly $\theta$-Hölder functions".

We will say that $f \in C^{n+\theta}\left(\mathbb{R}^{d} ; \mathbb{R}^{d}\right)$ ( $n \geq 1$ integer) if $f \in C^{\theta}\left(\mathbf{R}^{d} ; \mathbf{R}^{d}\right)$ and moreover the Fréchet derivatives $\mathcal{D}^{i} f(i=1, . ., n)$ are bounded and Hölder continuous of order $\theta \in(0,1)$. 


\section{The special transformation and the density of the solution}

Assume $k, d \geq 1$ and let $\left(B_{t}\right)_{t \in[0, T]}$ be a standard $k$-dimensional Brownian motion $\left(B=\left(B^{1}, \ldots, B^{k}\right)\right)$ on a probability space $(\Omega, \mathcal{F}, P)$ with respect to its natural filtration $\left(\mathcal{F}_{t}\right)_{t \geq 0}$. Consider the following stochastic differential equation in $\mathbb{R}^{d}$

$$
X_{t}(x)=x+\int_{0}^{t} b\left(X_{s}(x)\right) d s+\int_{0}^{t} \sigma\left(X_{s}(x)\right) d B_{s}, \quad t \in[0, T]
$$

or equivalently

$$
X_{t}(x)=x+\int_{0}^{t} b\left(X_{s}(x)\right) d s+\sum_{i=1}^{k} \int_{0}^{t} \sigma_{i}\left(X_{s}(x)\right) d B_{s}^{i}
$$

where $x \in \mathbb{R}^{d}$ and the stochastic integral in (5) is understood in the Itô sense.

As in [8], we shall assume throughout our work that the vector fields $b$ and $\sigma$ satisfy the following conditions

$$
b \in C^{\theta}\left(\mathbf{R}^{d} ; \mathbf{R}^{d}\right)
$$

and for every $i=1, . ., k$,

$$
\sigma_{i} \in C_{b}^{3}\left(\mathbf{R}^{d} ; \mathbf{R}^{d}\right)
$$

and $a=\sigma \sigma^{\star}\left(\sigma^{\star}\right.$ denotes the adjoint matrix of $\left.\sigma\right)$ is invertible and satisfies

$$
\|a\|_{0}=\sup _{x \in \mathbb{R}^{d}}\left\|a^{-1}(x)\right\|<\infty
$$

where for every $x \in \mathbb{R}^{d},\left\|a^{-1}(x)\right\|$ denotes the Hilbert-Schmidt norm of the $d \times d$ matrix $a^{-1}(x)$.

\subsection{The auxiliary elliptic system}

Fix $\lambda>0$ and fix $0<\alpha<\theta$ (recall that $\theta$ is fixed by condition (6) $)$ ). We introduce the auxiliary elliptic systems

$$
\lambda \psi_{\lambda}-L \psi_{\lambda}=b
$$

where $L$ is the Kolmogorov operator associated to (5) defined by

$$
L=\frac{1}{2} \operatorname{Tr}\left(\sigma \sigma^{\star} \mathcal{D}^{2}\right)+b(x) \mathcal{D} .
$$

We also introduce the function

$$
\phi_{\lambda}(x)=x+\psi_{\lambda}(x)
$$

where $\psi_{\lambda}$ is the unique classical solution in $C^{2+\alpha}\left(\mathbb{R}^{d} ; \mathbb{R}^{d}\right)$ of the equation (9)) (which exists due to Theorem 5 in [8]).

The following lemmas are due to [8] (see Theorem 5 and Lemma 8 in this reference) and they are key points for our method. Notice that we need to have $\sigma$ of class $C^{3}$ in order to have the second point below.

Lemma 1 For $\lambda>0$, let $\phi_{\lambda}$ be given by (11). Then the function $\phi_{\lambda}$ has the following properties when $\lambda$ is large enough: 
1. There exists $C>0$ such that $\left\|\mathcal{D} \phi_{\lambda}\right\|_{0}<C$.

2. $\phi_{\lambda}$ is a $C^{2}$-diffeomorphism.

3. $\phi_{\lambda}^{-1}$ has bounded first and second derivatives.

In the rest of the paper, we will consider $\lambda$ large enough in order that the point 1.-3. in Lemma 1 abovehold. The following special transform allows to remove the irregular drift in (5).

Lemma 2 We define the "new' coefficients $\widetilde{b}$ and $\widetilde{\sigma}$ from $b$ and $\sigma$ by

$$
\tilde{b}(x)=\lambda \psi_{\lambda}\left(\phi_{\lambda}^{-1}(x)\right) \text { and } \tilde{\sigma}(x)=\mathcal{D} \phi_{\lambda}\left(\phi_{\lambda}^{-1}(x)\right) \sigma\left(\phi_{\lambda}^{-1}(x)\right)
$$

for every $x \in \mathbb{R}^{d}$. Then

1. $\widetilde{b} \in C^{1+\alpha}\left(\mathbb{R}^{d} ; \mathbb{R}^{d}\right)$ and $\widetilde{\sigma} \in C^{1+\alpha}\left(\mathbb{R}^{d} ; \mathbb{R}^{d \times k}\right)$.

2. Consider the $S D E$

$$
Y_{t}(x)=y+\int_{0}^{t} \widetilde{b}\left(Y_{s}(x)\right) d s+\int_{0}^{t} \widetilde{\sigma}\left(Y_{s}(x)\right) d B_{s}, \quad t \in[0, T] .
$$

Then the SDE (13) is equivalent to (5) in the following sense: if $X$ solves (5), then $Y$ defined by

$$
\left.Y_{t}(x)=\phi_{\lambda}\left(X_{t}(x)\right)\right)
$$

solves (13) with $y=\phi_{\lambda}(x)$. The converse implication is also true.

The above two lemmas easily lead to the following result. Recall that a random variable in $\mathbb{R}^{d}$ is Malliavin differentiable if all its components are in $\mathbb{D}^{1,2}$.

Theorem 1 Assume (6), (7) and (8). Then, for every $t \in[0, T]$ and for every $x \in \mathbb{R}^{d}$, we have :

1. The random variable $X_{t}(x)$ is Malliavin differentiable.

2. The random variable $X_{t}(x)$ admits a density $\rho_{X_{t}(x)}$ with respect to the Lebesgue measure.

Proof: Recall that $Y$ solves the SDE (13) which is a SDE with smooth coefficients since $\widetilde{b}, \widetilde{\sigma} \in C^{1+\alpha}$, see Lemma 2. By standard arguments based on Malliavin calculus (see Chapter 2 in [22]) it follows that for every $t, x$, the random variable $Y_{t}(x)$ is Malliavin differentiable. Since $\phi_{\lambda}^{-1}$ is differentiable with bounded derivative by Lemma 1, we obtain the Malliavin differentiability of $X_{t}(x)$ for every $t, x$. Moreover, by applying the chain rule (3) , for every $r \in(0, T]$

$$
D_{r} X_{t}(x)=\mathcal{D} \phi_{\lambda}^{-1}\left(Y_{t}\right) D_{r} Y_{t}(x) .
$$

To show point 2., we note that $Y_{t}(x)$ admits a density with respect to the Lebesque measure. This can be argued by using Theorem 2.3.1 in [22], based on the fact that the diffusion matrix of $Y$ is non-singular, see Lemma 4 below. Denote by $\rho_{Y_{t}(x)}$ the density of the random variable $Y_{t}(x)$.

Next, for every continuous bounded function $\varphi: \mathbb{R}^{d} \rightarrow \mathbb{R}$, we can write, since $\phi_{\lambda}$ is a $C^{2}$ diffeomorphism, 


$$
\begin{aligned}
\mathbf{E} \varphi\left(X_{t}(x)\right) & =\mathbf{E} \varphi\left(\phi_{\lambda}^{-1}\left(Y_{t}(x)\right)\right) \\
& =\int_{\mathbb{R}^{d}} \varphi\left(\phi_{\lambda}^{-1}(u)\right) \rho_{Y_{t}(x)}(u) d u \\
& =\int_{\mathbb{R}^{d}} \varphi(u)\left(\operatorname{det} J \phi_{\lambda}(u)\right) \rho_{Y_{t}(x)}\left(\phi_{\lambda}(u)\right) d u
\end{aligned}
$$

where $J$ denotes the Jacobian (with respect to $x$ ). It follows from the last equality that for every $t>0$ and for every $x \in \mathbb{R}^{d}$ the random variable $X_{t}(x)$ admits a density $\rho_{X_{t}(x)}$ with respect to Lebesgue and the following formula holds true

$$
\rho_{X_{t}(x)}=\left(\operatorname{det} J \phi_{\lambda}\right) \cdot \rho_{Y_{t}(x)}\left(\phi_{\lambda}\right)
$$

Remark 1 Let us point out that in general (see e.g. [14], [15], [29]), proving the Malliavin differentiability of solutions to SDE with irregular drift demands pretty technical work.

It is also possible to give, in dimension 1, a representation of the density in the spirit of [21]. The following result is a consequence of Theorem 3.1 in [21].

Corollary 1 Assume $d=1$ and let the assumptions in Theorem 1 . Then, for every $t \in[0, T]$ and $x \in \mathbb{R}^{d}$, the density of the random variable $X_{t}(x)$ can be expressed as

$$
\rho_{X_{t}(x)}(z)=\frac{\mathbf{E}\left|X_{t}(x)\right|}{2 g_{X_{t}(x)}(z)} e^{-\int_{0}^{z} \frac{u}{g_{X_{t}(x)}(u)} d u}
$$

\section{Density of functions of the solution}

In this paragraph our aim is to prove the existence of densities for functions of the solution to SDEs with unbounded drift. Although the main idea comes from the special transformation (14), other auxiliary results are also neeeded.

We will first prove some auxiliary lemmas.

Lemma 3 Let $\phi_{\lambda}$ be given by (11). Then for every $x \in \mathbb{R}^{d}$ and for $\lambda$ large enough

$$
\left|\mathcal{D} \phi_{\lambda}(x)\right| \geq C>0 .
$$

Proof: From (11) we have for every $x \in \mathbb{R}^{d}$

$$
\mathcal{D} \phi_{\lambda}(x)=I_{d}+\mathcal{D} \psi_{\lambda}(x)
$$

where $I_{d}$ denoted the $d$-dimensional identity matrix. On the other hand, it follows from formula (18) in [8] that for every $x \in \mathbb{R}^{d}$

$$
\left|\mathcal{D} \psi_{\lambda}(x)\right| \leq c(\lambda)
$$

where $c(\lambda) \rightarrow 0$ as $\lambda \rightarrow \infty$. From (18), (19) and the triangle inequality, we get

$$
\begin{aligned}
\left|\mathcal{D} \phi_{\lambda}(x)\right| & =\left|I_{d}+\mathcal{D} \psi_{\lambda}(x)\right| \\
& \geq 1-\left|\mathcal{D} \psi_{\lambda}(x)\right| \geq C>0
\end{aligned}
$$

for $\lambda$ large enough. 
Lemma 4 Let $\tilde{\sigma}$ be given by (12). Then the diffusion matrix of the $S D E$ (13) $B=\tilde{\sigma} \widetilde{\sigma}^{*}$ is uniformly elliptic.

Proof: From the definition of the coefficient $\widetilde{\sigma}(12)$ we can write (recall that $a=\sigma \sigma^{*}$ ) for every $x \in \mathbb{R}^{d}$

$$
B(x)=\left(\mathcal{D} \phi_{\lambda}\right)\left(\phi_{\lambda}^{-1}\right) a\left(\phi_{\lambda}^{-1}\right)\left(\left(\mathcal{D} \phi_{\lambda}\right)\left(\phi_{\lambda}^{-1}\right)\right)^{*}
$$

and this implies that the inverse matrix of $B$ satisfies

$$
B^{-1}(x)=\left[\left(\left(\mathcal{D} \phi_{\lambda}\right)\left(\phi_{\lambda}^{-1}\right)\right)^{*}\right]^{-1} a^{-1}\left(\phi_{\lambda}^{-1}\right)\left(\left(\mathcal{D} \phi_{\lambda}\right)\left(\phi_{\lambda}^{-1}\right)\right)^{-1}
$$

Notice that, since $a^{-1}$ is bounded from (8) and by using the properties of $\phi_{\lambda}$ in Lemma 1, we immediately obtain the existence of a strictly positive constant $C$ such that

$$
\left|B^{-1}(x)\right| \leq C
$$

for every $x$.

Denote by $\|A\|$ the operator norm of the matrix $A$ and recall that if $A=A^{*}$ then

$$
\|A\|=\sup _{\lambda \in \operatorname{Spec}(A)}|\lambda|
$$

where $\operatorname{Spec}(A)$ is the spectrum of $A$. The above formula implies that

$$
\lambda_{i, B^{-1}}(x) \leq C
$$

for every $i \in I$ where $\operatorname{Spec}\left(B^{-1}\right)=\left\{\lambda_{i, B^{-1}}, i \in I\right\}$. Consequently,

$$
\lambda_{j, B}(x) \geq C
$$

for every $j \in J$ where we denoted the spectrum of $B$ by $\operatorname{Spec}(B)=\left\{\lambda_{j, B}, j \in J\right\}$. The above inequality (20) clearly gives

$$
\langle B(x) \xi, \xi\rangle \geq C|\xi|^{2} \text { for every } \xi \in \mathbb{R}^{d} .
$$

The converse inequality follows easily since $B$ is unifomly bounded and then

$$
\langle B(x) \xi, \xi\rangle \leq|B(x) \xi||\xi| \leq C|\xi|^{2} .
$$

Lemma 5 Let $Y$ be the solution to (13). Then for every $t \in[0, T], x \in \mathbb{R}^{d}$

$$
J Y_{t}(x)=e^{\int_{0}^{t}(\mathcal{D} \widetilde{\sigma})\left(Y_{s}(x)\right) d B_{s}+\int_{0}^{t}\left[(\mathcal{D} \widetilde{b})\left(Y_{s}(x)\right)-\frac{1}{2}(\mathcal{D} \widetilde{\sigma})^{2}\left(Y_{s}(x)\right)\right]}
$$

and

$$
J Y_{t}^{-1}(x)=e^{-\int_{0}^{t}(\mathcal{D} \widetilde{\sigma})\left(Y_{s}(x)\right) d B_{s}-\int_{0}^{t}\left[(\mathcal{D} \widetilde{b})\left(Y_{s}(x)\right)-\frac{1}{2}(\mathcal{D} \widetilde{\sigma})^{2}\left(Y_{s}(x)\right)\right]}
$$


Proof: Notice that the Jacobian of $Y$ satisfies the equation

$$
J Y_{t}(x)=1+\int_{0}^{t}(\mathcal{D} \widetilde{b})\left(Y_{s}(x)\right) Y_{s}(x) d s+\int_{0}^{t}(\mathcal{D} \widetilde{\sigma})\left(Y_{s}(x)\right) Y_{s}(x) d B_{s}
$$

while its inverse solves (see [22], page 126, formula (2.58)), for $r \in[0, T], x \in \mathbb{R}^{d}$,

$$
\left.J Y_{r}^{-1}(x)=1-\int_{0}^{r}(\mathcal{D} \widetilde{\sigma})\left(Y_{s}(x)\right)\left(J Y_{s}^{-1}(x)\right) d B_{s}-\int_{0}^{t}[\mathcal{D} \widetilde{b})\left(Y_{s}(x)\right)-(\mathcal{D} \widetilde{\sigma})^{2}\left(Y_{s}(x)\right)\right]\left(Y_{s}^{-1}(x)\right) d s .
$$

By solving the above two equations, we get the conclusion.

For simplicity, we will assume in the sequel that $d=k=1$. Consider a function $G$ : $[0, T] \times \mathbb{R} \rightarrow \mathbb{R}$ such that $G(t, \cdot) \in C_{b}^{1}(\mathbb{R})$ and

$$
\left|\partial_{x} G(t, x)\right| \geq C>0
$$

for every $t \in[0, T]$ and for every $x \in \mathbb{R}$, where $\partial_{x}$ denote the derivative with respect to the variable $x$.

The main result of this section states as follows.

Proposition 1 We assume (6), (7) and (8). Then, for every $t \in[0, T]$ and for every $x \in \mathbb{R}$, we have:

1. The random variable $G\left(t, X_{t}(x)\right)$ is Malliavin differentiable.

2. The random variable $G\left(t, X_{t}(x)\right)$ admits a density $\rho_{t, x}$ with respect to the Lebesgue measure.

Proof: Fix $t \in[0, T], x \in \mathbb{R}$. We have seen in Proposition 1 that the random variable $X_{t}(x)$ belongs to $\mathbb{D}^{1,2}$. Since $G$ is of class $C_{b}^{1}$ with respect to $x$, we get that $G\left(t, X_{t}(x)\right) \in \mathbb{D}^{1,2}$.

Moreover, for every $r>0$,

$$
\begin{aligned}
D_{r} G\left(t, X_{t}(x)\right) & =\partial_{x} G\left(t, X_{t}(x)\right) D_{r} X_{t}(x) \\
& =\partial_{x} G\left(t, X_{t}(x)\right) \mathcal{D} \phi_{\lambda}^{-1}\left(Y_{t}(x)\right) D_{r}\left(Y_{t}(x)\right)
\end{aligned}
$$

where we used relation (14) and we used twice the chain rule (3) for the Malliavin derivative.

On the other hand, from [22], formula (2.59) on page 126, we have

$$
D_{r} Y_{t}(x)=J Y_{t}(x)\left(J Y_{r}(x)\right)^{-1} \widetilde{\sigma}\left(Y_{r}(x)\right)
$$

where $\left(J Y_{t}(x)\right)^{-1}$ represents the inverse of $J Y_{t}(x)$ (recall that $Y$ generates a $C^{1}$ flow of diffeomorphism, see [8]). We thus get for $r \in(0, T]$

$$
D_{r} G\left(t, X_{t}(x)\right)=\partial_{x} G\left(t, X_{t}(x)\right) \mathcal{D} \phi_{\lambda}^{-1}\left(Y_{t}(x)\right) J Y_{t}(x)\left(J Y_{r}(x)\right)^{-1} \tilde{\sigma}\left(Y_{r}(t)\right) .
$$

and

$$
\begin{aligned}
\left\|D_{r} G\left(t, X_{t}(x)\right)\right\|_{L^{2}([0, T])}^{2} & =\int_{0}^{T}\left|\partial_{x} G\left(t, X_{t}(x)\right) \mathcal{D} \phi_{\lambda}^{-1}\left(Y_{t}(x)\right) J Y_{t}(x)\left(J Y_{r}(x)\right)^{-1} \tilde{\sigma}\left(Y_{r}(t)\right)\right|^{2} d r \\
& \geq C\left|J Y_{t}(x)\right|^{2} \int_{0}^{T}\left|\left(J Y_{r}(x)\right)^{-1}\right|^{2} d r
\end{aligned}
$$

where we used condition (21), Lemma 3 and Lemma 4 to bound from below $\partial_{x} G\left(t, X_{t}(x)\right), \mathcal{D} \phi_{\lambda}^{-1}\left(Y_{t}(x)\right)$ and $\widetilde{\sigma}\left(Y_{r}(t)\right)$ respectively. The result follows easily from Lemma 5 and the criterion (4). 
Remark 2 If $d \geq 1$, and the partial derivatives of $G:[0, T] \times \mathbb{R}^{d} \rightarrow \mathbb{R}$ with respect to $x \in \mathbb{R}^{d}$ are bounded (above and below) by strictly positive constants, then the above result can be also obtained. The formula (22) will become

$$
D_{r} G\left(t, X_{t}(x)\right)=\sum_{i=1}^{d} \frac{\partial G}{\partial x_{i}}\left(t, X_{t}(x)\right) \frac{\partial}{\partial x_{j}}\left(\phi_{\lambda}^{-1}\right)^{i}\left(Y_{t}(x)\right)\left(J Y_{t}(x)\right)_{j, l}\left(\left(J Y_{r}(x)\right)^{-1}\right)_{l k} \tilde{\sigma}^{k}\left(Y_{r}(x)\right)
$$

where the superscript means the components of functions and the subscripts are the elements of matrices. By using the boundedness of the partial derivatives of $G$ and Lemmas 3, ?? and 5, the strict positivity of $\left\|D_{r} G\left(t, X_{t}(x)\right)\right\|_{L^{2}([0, T])}^{2}$ can be proven.

Acknowledgement: The authors acknowledge partial support from the CNRS-FAPESP grant 267378. C. Olivera is partially supported by FAPESP by the grants $2017 / 17670-0$. and 2015/07278-0.

\section{References}

[1] O. Aboura and S. Bourguin (2013): Density estimates for solutions to one dimensional backward SDE's. Potential Anal. 38(2), 573-587.

[2] V. Bally and L. Caramellino, (2017): Regularity of probability laws by using an interpolation method. Ann. Probab., 45 (2), 1110-1159.

[3] V. Bally, L. Caramellino, R. Cont (2016): Stochastic integration by parts and functional It calculus. Lecture notes of the Barcelona Summer School on Stochastic Analysis held in Barcelona, July 2327, 2012. Advanced Courses in Mathematics. CRM Barcelona. Birkhuser/Springer,Cham.

[4] D. Baños and P. Krḧner (2017): Hölder continuous densities of solutions of SDEs with measurable and path dependent drift coefficients. Stochastic Process. Appl. 127 (6), 1785-1799.

[5] M. Besalú, A. Kohatsu-Higa and S. Tindel (2016): Gaussian type lower bounds for the density of solutions of SDEs driven by fractional Brownian motions. Ann. Probab. 44 (1), 399-443.

[6] A. Debussche and N. Fournier (2013): Existence of densities for stable-like driven SDEs with Hölder continuous coefficients, Journal of Functional Analysis 264, 1757-1778

[7] F. Flandoli, M. Gubinelli and E. Priola (2010). Well-posedness of the transport equation by stochastic perturbation. Invent. Math., 180(1): 1-53.

[8] F. Flandoli, M. Gubinelli, E. Priola (2010): Flow of diffeomorphisms for SDEs with unbounded Hölder continuous drift. Bulletin des Sciences Mathematiques, 134, 405-422.

[9] A. Friedman (1975): Stochastic Differential Equations and Applications. New York, Academic Press, 1975.

[10] A. Friedman (1964): Partial differential equations of parabolic type. Prentice Hall, Engle- wood Cliffs, NJ.

[11] N. Fournier and J. Printems (2010): Absolute continuity for some one-dimension al processes. Bernoulli, 16(2), 343-360. 
[12] M. Hayashi, A. Kohatsu-Higa and G. Yk̂̀i (2013) : Local Hlder continuity property of the Densities of Solutions of SDEs with Singular Coefficients. J. Theoret. Probab., 26(4), 1117-1134.

[13] A. Kohatsu-Higa, (2003): Lower bounds for densities of uniformly elliptic ran- dom variables on Wiener space. Probability Theory and Related Fields 126, 421-457.

[14] A. Kohatsu-Higa and A. Tanaka (2012): Malliavin Calculus method to study densities of additive functionals of SDE's with irregular drifts. Ann. Inst. Henri Poincar Probab. Stat. 48(3), 871-883.

[15] A. Kohatsu-Higa and A. Makhlouf (2013): Estimates for the density of functionals of sdes with irregular drift. Stochastic Processes and their Applications 123(5), 1716-1728.

[16] H. Kunita (1984): Stochastic differential equations and stochastic flows of diffeomorphisms. Ecole d'été de probabilités de Saint-Flour, XII1982, 143303, Lecture Notes in Math., 1097, Springer, Berlin.

[17] H. Kunita (1984): First Order Stochastic Partial Differential Equations, in Proceedings of the Taniguchi International Symposium on Stochastic Analysis, North-Holland Mathematical Library, 249-269.

[18] O. A. Ladyenskaja, V. A. Solonnikov and N. N. Uralceva (1968): Linear and Quasilinear Equations of Parabolic Type. Translations of Mathematical Monographs 23. Amer. Math. Soc., Providence, RI.

[19] S. De Marco. (2011): Smoothness and asymptotic estimates of densities for SDEs with locally smooth coefficients and applications to square root-type diffusions. Ann. Appl. Probab., 21(4), 1282-1321 .

[20] I. Nourdin and G. Peccati (2012): Normal Approximations with Malliavin Calculus From Stein's Method to Universality. Cambridge University Press.

[21] I. Nourdin and F. Viens (2009): Density formula and concentration inequalities with Malliavin calculus. Electronic Journal of Probability, 14, paper 78, 2287-2309.

[22] D. Nualart (2006): Malliavin Calculus and Related Topics. Second Edition. Springer New York.

[23] D. Nualart and L. Quer-Sardanyons (2009): Gaussian density estimates for solutions to quasilinear stochastic partial differential equations. Stochastic Process. Appl. 119(11), 3914-3938.

[24] M. Romito (2016): Time regularity of the densities for the NavierStokes equations with noise. Journal of Evolution Equations,16(3), 503-518.

[25] M. Romito (2017): A simple method for the existence of a density for stochastic evolutions with rough coefficients. Preprint, arXiv:1707.05042.

[26] M. Sanz-Solé (1995): Malliavin Calculus. With applications to stochastic partial differential equations. Fundamental Sciences, EPFL Press, Lausanne.

[27] I. Shigekawa (1980): Derivatives of Wiener functionals and absolute continuity of induced measures. J. Math. Kyoto Univ. 20(2), 263-289.

[28] D. Stroock (1988): Diffusion semigroups corresponding to uniformly elliptic divergence form operators. In Séminaire de probabilités de Strasbourg XXII 316347. Springer, Berlin. 
[29] X. Zhang (2014): Stochastic differential equations with Sobolev diffusion and singular drift. Ann. Appl. Probab. 26 (5), 2697-2732. 